\title{
CLASSICAL SIGNAL MODEL FOR QUANTUM CHANNELS
}

\author{
Andrei Khrennikov \\ nternational Center for Mathematical Modelling \\ in Physics and Cognitive Sciences, \\ Linnaeus University, Växjö, S-35195, Sweden \\ Masanori Ohya and Naboru Watanabe \\ Department of Information Sciences, Tokyo University of Science \\ Yamasaki 2641, Noda-shi, Chiba, 278-8510 Japan
}

\begin{abstract}
Recently it was shown that the main distinguishing features of quantum mechanics (QM) can be reproduced by a model based on classical random fields, so called prequantum classical statistical field theory (PCSFT). This model provides a possibility to represent averages of quantum observables, including correlations of observables on subsystems of a composite system (e.g., entangled systems), as averages with respect to fluctuations of classical (Gaussian) random fields. In this note we consider some consequences of PCSFT for quantum information theory. They are based on the observation [1] of two authors of this paper that classical Gaussian channels (important in classical signal theory) can be represented as quantum channels. Now we show that quantum channels can be represented as classical linear transformations of classical Gaussian signals.
\end{abstract}

\section{Introduction}

At the very beginning of QM the idea that quantum mechanics is simply a special model of wave mechanics was quite popular. It was supported 
by the discovery of "quantum wave equation" by Schrödinger and by the association with each particle its wave-length, the De Broglie wave-length. However, both Schrödinger and De Broglie should give up in front of the difficulties of the interpretation of "quantum waves" as real physical waves, see [2] for debates. The main problem of the "physical wave interpretation" was the impossibility to describe a composite system by waves defined on the physical space, $X=\mathbf{R}^{3}$. The wave function of a composite system is defined on the space $X_{m}=\mathbf{R}^{3 m}$, where $m$ is the number of subsystems. Nevertheless, nowadays various wave models are popular in attempts to go beyond QM. At the present time the most successful models are stochastic electrodynamics (SED) [3], and semiclassical theory [4].

Recently [5]- [7] it was shown that the main distinguishing features of quantum mechanics (QM) can be reproduced by a model based on classical random fields, so called prequantum classical statistical field theory (PCSFT). This model provides a possibility to represent averages of quantum observables, including correlations of observables on subsystems of a composite system (e.g., entangled systems), as averages with respect to fluctuations of classical (Gaussian) random fields. In this note we consider some consequences of PCSFT for quantum information theory. They are based on the observation [1] of two authors of this paper that classical Gaussian channels (important in classical signal theory) can be represented as quantum channels. Now we show that quantum channels can be represented as classical linear transformations of classical Gaussian signals.

Finally, we mention another mathematical model reducing quantum randomness to classical one, namely, tomographic approach, see, e.g., 8]. It is an interesting (but may be quite complicated) problem to analyze the interplay between quantum and classical information theories in this approach.

\section{Brief presentation of PCSFT}

\subsection{Classical fields as hidden variables}

Classical fields are selected as hidden variables. Mathematically these are functions $\phi: \mathbf{R}^{3} \rightarrow \mathbf{C}$ (or more generally $\rightarrow \mathbf{C}^{k}$ ) which are square integrable, i.e., elements of the $L_{2}$-space.

The latter condition is standard in the classical signal theory; in particular, for the electromagnetic field this is just the condition that the energy is 
finite

$$
\mathcal{E}(\phi)=\int_{\mathbf{R}^{3}}\left(E^{2}(x)+B^{2}(x)\right) d x<\infty
$$

or by using is the Riemann-Silbertstein vector $\phi(x)=E(x)+i B(x):\|\phi\|^{2}=$ $\int_{\mathbf{R}^{3}}|\phi(x)|^{2}<\infty$. Thus the state space of our prequantum model is $H=$ $L_{2}\left(\mathbf{R}^{3} ; \mathbf{C}\right)$, the space of square integrable complex valued fields on "physical space" $\mathbf{R}^{3}$. Formally, the same state space is used in QM, but we prefer to emphasize coupling with the classical signal theory. For example, the quantum wave function should satisfy the normalization condition $\|\psi\|^{2}=1$, but a PCSFT-state can be any vector of $H$.

A random field (at the fixed instant of time) is a function $\phi(x, \omega)$, where $\omega$ is the random parameter. Thus, for each $\omega_{0}$, we obtain the classical field, $x \mapsto \phi\left(x, \omega_{0}\right)$. Another picture of the random field is the $H$-valued random variable, each $\omega_{0}$ determines a vector $\phi(\omega) \in H$. A random field is given by a probability distribution on $H$. To simplify considerations, we can consider a finite-dimensional Hilbert space, instead of $L_{2}\left(\mathbf{R}^{3} ; \mathbf{C}\right.$ ) (as people often do in quantum information theory). In this case our story is on $H$-valued random vectors, where $H=\mathbf{C}^{n}$. This is the ensemble model of the random field. In the rigorous mathematical framework it is based on Kolmogorov probability space $(\Omega, \mathcal{F}, \mathbf{P})$, where $\Omega$ is a set and $\mathcal{F}$ is a $\sigma$-algebra of its subsets, $\mathbf{P}$ is a probability measure on $\mathcal{F}$. As is well known from the classical signal theory, one can move from the ensemble description of randomness to the time series description - under the ergodicity hypothesis.

By applying a linear functional $y$ to the random vector $\phi$ we obtain the scalar random variable. In the $L_{2}$-case we get a family of scalar random variables:

$$
\omega \mapsto \xi_{y}(\omega) \equiv \int y(x) \overline{\phi(x, \omega)} d x, y \in L_{2} .
$$

We recall that the covariance operator $D$ of a random field (with zero average) $\phi \equiv \phi(x, \omega)$ is defined by its bilinear form:

$$
\langle D u, v\rangle=E\langle u, \phi\rangle\langle\phi, v\rangle, u, v \in H .
$$

Under the additional assumption that the prequantum random fields are Gaussian, the covariance operator uniquely determines the field. We shall poceed under the assumption that prequantum fields are Gaussian. We also suppose that that all prequantum fields have zero average:

$$
E\langle y, \phi\rangle=0, y \in H
$$


where $E$ denotes the classical mathematical expectation (average, mean value). If $H=\mathbf{C}^{n}$, where $\phi(\omega)=\left(\phi_{1}(\omega), \ldots, \phi_{n}(\omega)\right)$, then condition (3) , zero average, is reduced to $E \phi_{i}=0, i=1,2$, and the covariance matrix $D=\left(d_{k l}\right)$, where $d_{k l}=E \phi_{k} \bar{\phi}_{l}$.

\subsection{Covariance operator interpretation of wave func- tion}

In our model the wave function $\psi$ of the QM-formalism encodes a prequantum random field: $\phi \equiv \phi_{\psi}$. The QM-terminology, "a quantum system in the state $\psi$ ", is translated into the PCSFT-terminology, a "random field." In PCSFT the $\psi$-function determines the covariance operator of the prequantum random field. For simplicity, we consider the case of a single, i.e., noncomposite, system, e.g., photon or electron. In this situation normalization (by dispersion) of the covariance operator $D$ of the prequantum field is given by the orthogonal projector on the vector $\psi$ (the density operator corresponding to this pure state)

$$
\rho_{\psi}=\psi \otimes \psi
$$

i.e., $\rho_{\psi} u=\langle u, \psi\rangle \psi, u \in H$. The covariance operator of the prequantum field is given by

$$
D=\sigma^{2}(\phi) \rho_{\psi},
$$

where

$$
\sigma^{2}(\phi)=E\|\phi\|^{2}(\omega)=\operatorname{TrD}
$$

is the dispersion of the prequantum random field $\phi \sim N(0, D)$. To determine the covariance operator $D$ on the basis of the density operator $\rho_{\psi}$, one should determine the scale of fluctuations of the prequantum field given by the dispersion $\sigma^{2}(\phi)$.

\subsection{Quantum observables from quadratic forms of the prequantum field}

In PCSFT quantum observables are represented by corresponding quadratic

forms of the prequantum field. A self-adjoint operator $\widehat{A}$ is considered as the symbolic representation of the PCSFT-variable:

$$
\phi \mapsto f_{A}(\phi)=\langle\widehat{A} \phi, \phi\rangle .
$$


We remark that $f_{A}$ can be considered as a function on the phase space of classical fields: $f_{A} \equiv f_{A}(q, p)$, where $\phi(x)=q(x)+i p(x), q, p \in L_{2}\left(\mathbf{R}^{3} ; \mathbf{R}\right)$, the space of real valued fields.

Consider the quantum average

$$
\left\langle f_{A}\right\rangle_{\mathrm{QM}}=\langle\widehat{A} \psi, \psi\rangle
$$

and the classical average

$$
\left\langle f_{A}\right\rangle_{\mathrm{CL}}=E f_{A}(\phi)
$$

They coincide up to a scaling factor

$$
\left\langle f_{A}\right\rangle_{\mathrm{CL}}=\operatorname{TrD}\left\langle\mathrm{f}_{\mathrm{A}}\right\rangle_{\mathrm{QM}}
$$

In the real physical case $H$ is infinite-dimensional; the classical average is given by the integral over all possible classical fields; probabilistic weights of the fields are determined by the $\psi$.

\subsection{Quantum and prequantum interpretations of Schrödinger's equation}

Main message: Schrödinger's equation does not describe the dynamics of a wave of probability. The same equation plays a double role. On the one hand, it describes dynamics of a physical random field. On the other hand, it encodes the dynamics of the covariance operator of this field.

Before to go to the PCSFT-dynamics, we consider the Schrödinger equation in the standard QM-formalism:

$$
\begin{aligned}
i h \frac{\partial \psi}{\partial t}(t, x) & =\widehat{\mathcal{H}} \psi(t, x), \\
\psi\left(t_{0}, x\right) & =\psi_{0}(x),
\end{aligned}
$$

where $\widehat{\mathcal{H}}$ is Hamiltonian, the energy observable. Although Shcrödinger tried to interpret $\psi(t, x)$ as a classical field (e.g., the electron field), the conventional interpretation is the probabilistic one, due to Max Born.

We recall that a time dependent random field $\phi(t, x, \omega)$ is called the stochastic process (with the state space $H$ ). The dynamics of the prequantum random field is described by the simplest stochastic process which is given by deterministic dynamics with random initial conditions. 
In PCSFT the Schrödinger equation, but with the random initial condition, describes the dynamics of the prequantum random field, i.e., the prequantum stochastic process can be obtained from the same mathematical equation as it was used in QM for the dynamics of the wave function:

$$
\begin{aligned}
i h \frac{\partial \phi}{\partial t}(t, x, \omega) & =\widehat{\mathcal{H}} \phi(t, x, \omega), \\
\phi\left(t_{0}, x, \omega\right) & =\phi_{0}(x, \omega),
\end{aligned}
$$

where the initial random field $\phi_{0}(x, \omega)$ is determined by the quantum pure state $\psi_{0} \sim N(0, D)$, where $D$ is given by (5). Hence, the standard QM provides the knowledge of the covariance operator of the prequantum random field.

The PCSFT dynamics (13), (14) matches with the standard QM-dynamics (11), (12) - by taking into account the PCSFT-interpretation of the wavefunction, see (4). Set

$$
\rho(t)=D(t) / \operatorname{Tr} \mathrm{D}(\mathrm{t}),
$$

where $D(t)$ is the covariance operator of the random field $\phi(t) \equiv \phi(t, x, \omega)$, the solution of (13), (14). Then

$$
\rho(t) \equiv \rho_{\psi}(t)=\psi(t) \otimes \psi(t)
$$

where $\psi(t)$ is a solution of (11), (12).

\subsection{Random fields corresponding to mixed states}

We now consider the general quantum state given by a density operator $\rho$. By PCSFT it is determined as normalization of the covariance operator of the corresponding prequantum field

$$
\rho=D / \operatorname{Tr} \mathrm{D}
$$

The dynamics of the prequantum field $\phi(t, x, \omega)$ is also described by the Shrödinger equation, see (13), (14), with the random initial condition $\phi_{0}(x, \omega) \sim$ $N(0, D)$. 


\section{Linear transformations of Gaussian random fields}

Let $H_{i}, i=1,2$, be complex Hilbert spaces. Consider a linear bounded operator $V: H_{1} \rightarrow H_{2}$ (we remark that $V^{*}: H_{2} \rightarrow H_{1}$ ) and the corresponding linear filter

$$
\phi_{\text {out }}(\omega)=V \phi_{\text {in }}(\omega),
$$

where $\phi_{\text {in }}$ is the $H_{1}$-valued random field which is ditributed $N\left(0, D_{\text {in }}\right)$. Then the $H_{2}$-valued random field $\phi_{\text {out }} \sim N\left(0, D_{\text {out }}\right)$, where

$$
D_{\text {out }}=V D_{\text {in }} V^{*}
$$

Example 1. (Unitary evolution) The solution of the Schrödinger equation (13) with the initial condition $\phi_{\text {in }}$, a Gaussian random field, can be represented in the form of the linear filter (16), where $V=U_{t}=\exp \{-i t \widehat{\mathcal{H}} / h\}$. This filter preserves the norm of the prequantum field

$$
\left\|\phi_{\text {out }}(t, \omega)\right\|^{2}=\left\|\phi_{\text {in }}(\omega)\right\|^{2} .
$$

Hence, this filter preserves even the dispersion of a classical signal

$$
\sigma^{2}\left(\phi_{\text {out }}\right)(t)=\operatorname{Tr}_{\text {out }}(\mathrm{t})=\sigma^{2}\left(\phi_{\text {in }}\right)=\operatorname{Tr}_{\text {in }} .
$$

Example 2. (von Neumann-Lüders projection). Let $L=P$ be an orthogonal projector $P: H \rightarrow E$, where $E$ is a linear subspace of $H$. Here the $E$-valued (Gaussian) random field $\phi_{\text {out }}(\omega)=P \phi_{\text {in }}(\omega)$ describes the output after the von Neumann-Lüders projection-measurement (with filtration with respect to the value corresponding to the projector $P$ ). The the von Neumann-Lüders filter does not preserve even the dispersion

$$
\operatorname{TrD}_{\text {out }}=\operatorname{TrPD}_{\text {in }} \mathrm{P} \neq \operatorname{TrD}_{\text {in }}
$$

Moreover,

$$
\operatorname{Tr} \mathrm{D}_{\text {out }} \leq \operatorname{TrD}_{\text {in }},
$$

i.e., the dispersion of a signal always decreases.

Example 3. (von Neumann-Lüders measurement). Consider a dichotomous quantum observable $\widehat{A}$ with eigenvalues $\alpha_{1}$ and $\alpha_{2}$ and eigensubspaces 
$E_{1}$ and $E_{2}$. Denote corresponding projectors by $P_{1}$ and $P_{2}$. They are orthogonal. Take two independent random fields $\phi_{k} \sim N(0, D), k=1,2$, where the corresponding quantum state $\rho=D / \operatorname{TrD}$. We form the $H \times H$ valued random variable $\phi_{\text {in }}(\omega)=\left(\phi_{1}(\omega), \phi_{2}(\omega)\right) \sim N(0, D \times D)$. We define a linear operator $V: H \times H \rightarrow H, V(x, y)=P_{1} x+P_{2} y$ and the corresponding linear filter

$$
\phi_{\text {out }}(\omega)=V \phi_{\text {in }}(\omega)=P_{1} \phi_{1}(\omega)+P_{2} \phi_{2}(\omega) .
$$

Since $\phi_{1}$ and $\phi_{2}$ are independent and Gaussian, their linear transforms $P_{1} \phi_{1}$ and $P_{2} \phi_{2}$ are also independent. Hence, the covariance operator of the random field $\phi_{\text {out }}$ equals to the sum of covariance operators of the latter two random fields, i.e.,

$$
D_{\text {out }}=P_{1} D_{\text {in }} P_{1}+P_{2} D_{\text {in }} P_{2} .
$$

We remark that the dispersion is preserved

$$
\operatorname{Tr} \mathrm{D}_{\text {out }}=\operatorname{TrD}_{\text {in }}
$$

This example can be easily generalized to an arbitrary quantum observables with purely discrete spectrum, $\widehat{A}=\sum_{k} \alpha_{k} P_{k}$; moreover, to any POVM. For simplicity, we present the case of measurement with finitely many results.

Example 3. (POVM-measurement as classical linear filter) Consider a $\operatorname{POVM}\left\{Q_{i}=V_{i} V_{i}^{*}\right\}_{i=1}^{n}$, where

$$
\sum_{i=1}^{n} Q_{i}=I
$$

Define the corresponding linear map $V: H_{1} \times \ldots \times H_{n} \rightarrow H, V\left(x_{1}, \ldots, x_{n}\right)=$ $V_{1} x_{1}+\ldots+V_{n} x_{n}$. Consider also a quantum state, density operator $\rho$. Take a vector $\phi_{\text {in }}(\omega)=\left(\phi_{1}(\omega), \ldots, \phi_{n}(\omega)\right)$ consisting of equaly distributed independent Gaussian random fields of $N(0, D)$-type, where the quantum state under consideration $\rho=D / \operatorname{TrD}$. We define a linear filter corresponding to the map $V$

$$
\phi_{\text {out }}(\omega)=V \phi_{\text {in }}(\omega)=\sum_{i} V_{i} \phi_{i}(\omega)
$$

We have

$$
D_{\text {out }}=\sum_{i} V_{i} D_{\text {in }} V_{i}^{*} .
$$


This is nothing else than a completely positive map, see, e.g., [9]. Hence, it preserves the trace, i.e., the equality (22) holds. Therefore the equality (25) for covariance operators is transformed into the equality for quantum states, density operators

$$
\rho_{\text {out }}=\frac{D_{\text {out }}}{\operatorname{Tr} D_{\text {out }}}=\frac{D_{\text {out }}}{\operatorname{TrD}_{\text {in }}}=\sum_{i} \frac{\operatorname{Tr} \mathrm{V}_{\mathrm{i}} \mathrm{D}_{\text {in }} \mathrm{V}_{\mathrm{i}}^{*}}{\operatorname{Tr} \mathrm{D}_{\text {in }}} \rho_{\text {out }, \mathrm{i}},
$$

where

$$
\rho_{\text {out }, \mathrm{i}}=\frac{V_{i} D_{\text {in }} V_{i}^{*}}{\operatorname{Tr}_{\mathrm{i}} \mathrm{D}_{\mathrm{in}} \mathrm{V}_{\mathrm{i}}^{*}}
$$

Set $\rho_{\text {in }}=D_{\text {in }} / \operatorname{Tr} D_{\text {in }}$. Then

$$
\rho_{\text {out }, \mathrm{i}}=\frac{V_{i} \rho_{\text {in }} V_{i}^{*}}{\operatorname{Tr} V_{\mathrm{i}} \rho_{\text {in }} V_{\mathrm{i}}^{*}}
$$

and

$$
\rho_{\text {out }}=\sum_{i}\left(\operatorname{TrV}_{\mathrm{i}} \rho_{\text {in }} \mathrm{V}_{\mathrm{i}}^{*}\right) \rho_{\text {out }, \mathrm{i}}
$$

Since any quantum channel can represented (the Kraus representation, [9]) in the form (25), in general with the infinite number of terms, we demonstrated (generalization to the infinite number of terms in the Kraus decomposition is straightforward) that any quantum channel can be represented as the linear filter of classical signals of the form (24). This is a step in the same direction as paper [1]: exploring the analogy between quantum information theory and classical signal theory.

This research project on the interplay between classical and quantum information theory started during the visiting fellowship of A. Khrennikov at Tokyo University of Science, March 2010, which was supported by the grant QBIC.

\section{References}

[1] M. Ohya and N. Watanabe, Jap. J. Appl. Math., 3, 197 (1986).

[2] L. Accardi, G. Adenier, C. Fuchs, G. Jaeger, A. Khrennikov, J.-A. Larsson, S. Stenholm (eds), Foundations of Probability and Physics-5, Ser. Conferfence Proceedings, American Institute of Physics, Melville, NY, 1101 (2009). 
[3] L. De la Pena and A. Cetto, The Quantum Dice: An Introduction to Stochastic Electrodynamics, Kluwer, Dordrecht (1996).

[4] M. O. Scully and M. S. Zubairy, Quantum Optics, Cambridge University Press, Cambridge (1997).

[5] A. Khrennikov, J. Phys. A: Math. Gen., 38, 9051 (2005).

[6] A. Khrennikov, EPL, 88, 40005 (2009).

[7] A. Khrennikov, J. of Russian Laser Research, 31, 101-209 (2010).

[8] V. I. Manko, J. of Russian Laser Research, 17, 579 (1996).

[9] R. S. Ingarden, A. Kossakowski, and M. Ohya, Information Dynamics and Open Systems: Classical and Quantum Approach, Kluwer, Dordrecht (1997). 